\title{
Vulnerabilidad Social en el Síndrome de Turner: Interacción Genes-Ambiente
}

Vulnerabilidade Social na Síndrome de Turner: Interação Genes-Ambiente

Social Vulnerability in Turner's Syndrome: Genes-Environment Interactions
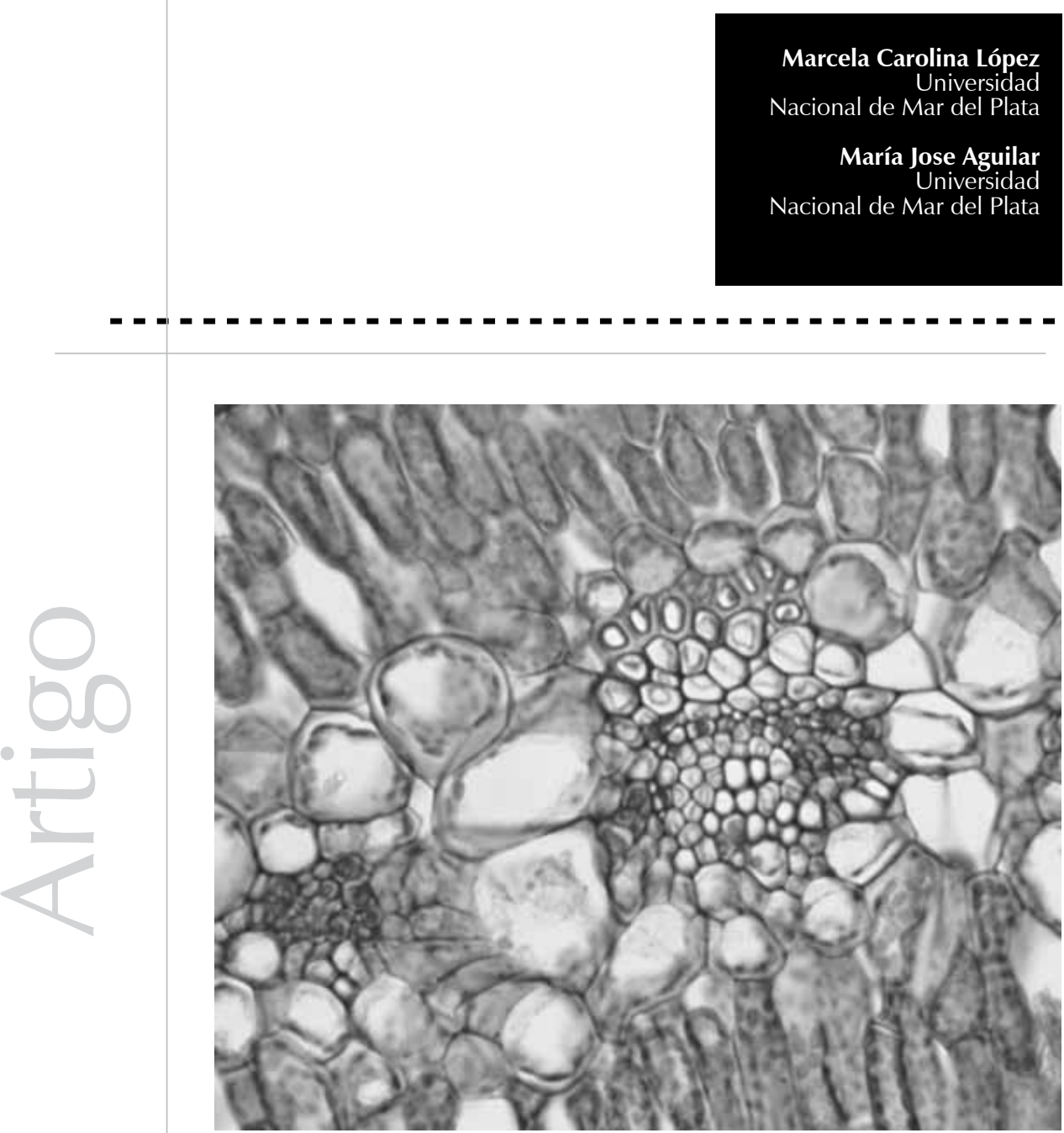
Resumen: En este trabajo se exponen los fundamentos teóricos que permiten considerar al Síndrome de Turner como un "modelo genético sensible" en el cual analizar asociaciones entre caracteres psicológicos y factores genéticos y ambientales. La investigación en esta línea permitiría dilucidar mecanismos de riesgo ambiental y comprender el grado de "vulnerabilidad" que presentan las mujeres con el síndrome. El Síndrome de Turner es un trastorno genético, no heredable, que se caracteriza por la deleción total o parcial del cromosoma $X$ en el sexo femenino. El estudio de la disfunción social que presentan las mujeres con este síndrome, es decir, de la capacidad para relacionarse con el entorno y la calidad de los vínculos tiene importantes implicaciones en la comprensión de la forma en que actúa el ambiente sobre el desarrollo psicológico, y de cómo a su vez éste se ve afectado por factores genéticos que actúan en interacción.

Palabras claves: Síndrome de Turner. Interacción genes-ambiente. Riesgo ambiental. Disfunción social.

Resumo: O trabalho apresenta base teórica que permite considerar a síndrome de Turner como um "modelo genético sensível" no qual é possível analisar associações entre caraterísticas psicológicas e fatores genéticos e ambientais. A pesquisa nessa linha permitiria desvendar mecanismos de risco ambiental e compreender o grau de "vulnerabilidade" que as mulheres com a síndrome apresentam. A síndrome de Turner é um transtorno genético não hereditário, produto da perda total ou parcial do cromossomo X no sexo feminino. O estudo da disfunção social que as mulheres com essa síndrome apresentam é importante para a compreensão da forma como atua o ambiente sobre o desenvolvimento psicológico, e de como, por sua vez, é afetado por fatores genéticos que atuam em interação.

Palavras-chave: Síndrome de Turner. Interação genes-ambiente. Risco ambiental. Disfunção social.

Abstract: In this work we describe the theoretical foundations that allow consider the Turner's syndrome as a "sensitive genetic model" in which it is possible to analyze associations between psychological characteristics and genetic and environmental factors. Research in this area would elucidate mechanisms of environmental risk and permit to understand the degree of "vulnerability" that women with the syndrome present. The Turner syndrome is a non-inherited genetic disorder, characterized by total or partial deletion of the chromosome $X$ in females. The study of the social dysfunction that women with this syndrome present, that is, the ability to interact with the environment and the quality of the links has important implications in understanding how the environment acts on psychological development and how this in turn is affected by the genetic factors that operate in interaction.

Keywords: Turner's Syndrome. Genes-Environment interaction. Environment risk. Social dysfunction.

\section{Riesgo ambiental en poblaciones genéticamente sensibles}

El dilema entre herencia y ambiente ha marcado gran parte de la historia de la Psicología generando fuertes polémicas dentro de este campo. Durante el siglo pasado, ambientalistas e innatistas trataron de demostrar el predominio de un u otro aspecto en el desarrollo humano. Sin embargo, en el presente, la mayoría de las investigaciones que intentan comprender los procesos involucrados en el desarrollo requieren modelos relacionales, interactivos, donde los factores genéticos y ambientales aparecen operando en conjunción (Bleichmar, 2004).
Actualmente, la Genética del comportamiento es, sin lugar a dudas, uno de los principales campos de investigación psicológica. Los avances científicos producidos en los últimos años en las disciplinas de Genética cuantitativa y molecular se han dado cuenta de que los genes juegan un papel importante en la conducta humana, aportando, asimismo, la mejor evidencia disponible sobre la importancia del ambiente en las diferencias individuales de los caracteres psicológicos. En este sentido los aportes más interesantes al estudio del ambiente en el campo de la Genética del comportamiento 
están relacionados con tres aspectos: el impacto de las influencias ambientales no compartidas, las correlaciones entre los genes y el ambiente, y las interacciones genotipoambiente (Plomin, DeFries, McClearn, \& McGuffin, 2002). Este último aspecto se refiere a la sensibilidad genética de los individuos a determinadas condiciones ambientales, uno de los principales objetivos dentro de este campo de investigación es identificar factores de riesgo ambiental en individuos genéticamente sensibles.

En el presente trabajo se exponen los fundamentos teóricos que permiten considerar al Síndrome de Turner como un "modelo genético sensible" en el cual analizar asociaciones entre caracteres psicológicos y factores genéticos y ambientales. La investigación en esta línea permitiría dilucidar mecanismos de riesgo ambiental y comprender el grado de "vulnerabilidad" que presentan las mujeres con el síndrome. Esta vía de estudio síndrome-gen-conducta proporciona resultados mas rápidos que otros niveles de análisis en términos de predicción, intervención y prevención (Artigas Pallarés, Gabau Vila, \& Guitart Feliubadaló, 2006).

\section{Comprendiendo las contribuciones relativas de los genes y el ambiente al fenotipo}

El análisis genético clásico se centra en caracteres que están determinados por la variabilidad alélica de un único gen, presentando un modo mendeliano simple de transmisión, donde la posibilidad de expresión fenotípica queda definida por la presencia de un determinado par de alelos. Sin embargo, la mayoría de los rasgos de interés para la Psicología son poligénicos o multifactoriales, es decir, son el resultado de la interacción y correlación entre varios pares de genes y el ambiente. A diferencia de los caracteres de localización única, los rasgos multigénicos no poseen una herencia sencilla, identificable, resultando complejo analizar las contribuciones relativas de los genes y el ambiente al fenotipo (Kandel, 2000).

La interacción entre determinados genes y ambientes confiere a los individuos diferentes grados de susceptibilidad en la aparición de una característica determinada. El rasgo aparece en individuos que han alcanzado un determinado umbral, así la variabilidad en la expresión fenotípica podría estar relacionada con el mayor o menor número de genes predisponentes en cada individuo. La expresión diferencial de los genes dependiendo del ambiente se denomina norma de reacción, y alude a genes que pueden expresarse de una manera diferente en distintos individuos, dependiendo del ambiente en que esos individuos se desarrollen. La norma de reacción es el conjunto de vías que puede seguir un genotipo, en todos los ambientes favorables y desfavorables, hasta dar lugar a los diversos fenotipos posibles (Puertas, 1992).

Lo anterior da cuenta que un gen no determina un fenotipo actuando aisladamente, sino en relación con el ambiente $y$, en muchas ocasiones, con otros genes del mismo individuo a lo largo de la embriogénesis (Molenaar, Boomsma, \& Dolan, 1993). Este conjunto de factores puede, en ocasiones, hacer que para un mismo genotipo pueda existir una amplia variabilidad fenotípica. Para comprender esto último es necesario 
explicar algunos conceptos que marcan parte de estas relaciones, en el caso del Síndrome de Turner son: mosaicismo, impronta genómica, penetrancia y expresividad. Mosaicismo corresponde a la coexistencia, en un mismo individuo, de dos o más poblaciones celulares con distinto genotipo. El fenómeno de impronta hace referencia a la expresión diferencial de los genes según sean de procedencia paterna o materna. La penetrancia se define como el porcentaje de individuos que presentan el fenotipo correspondiente a un determinado genotipo, es la frecuencia con que se expresa ese genotipo. Por último, la expresividad, es el porcentaje de individuos que muestran el fenotipo completo de un rasgo en relación con aquellos que poseen el mismo genotipo pero lo expresan parcialmente, hace referencia a la variabilidad de un rasgo heredado (Suzuki, Griffiths, Miller, \& Lewontin, 1996).

Cabe considerar que estos mismos efectos podrían deberse también a la influencia de factores de naturaleza ambiental. En Genética cuantitativa la palabra "ambiente" incluye todas las influencias excepto la herencia, esto implica un uso mucho más amplio del que se hace en Psicología. Según esta definición, el ambiente abarca acontecimientos prenatales y circunstancias biológicas como la nutrición o la enfermedad, no solamente los factores familiares y los relativos a la socialización (Plomin \& Crabbe, 2000).

1 La heredabilidad es un estadístico poblacional que estima que proporción de las diferencias fenotípicas observadas se debe a diferencias genéticas. estrategias de dos disciplinas: la Genética molecular y la Genética cuantitativa. Los avances científicos en Genética molecular han permitido el conocimiento de la secuencia completa del genoma humano, facilitando la localización e identificación de genes, sin embargo aún no se conoce la totalidad de proteínas codificadas por los mismos y la relación entre éstas y el comportamiento humano. El avance científico en este sentido aportará marcadores de ADN para identificar genotipos medibles, que se utilizarán para comprender las vías que conectan los genes y la conducta. Hasta el momento en que se pueda descifrar cómo operan los genes Plomin, Owen y McGuffin (2004) proponen utilizar genotipos conocidos, especialmente los trastornos genéticos, como modelos de estudio.

La Genética cuantitativa determina, a través de estudios poblacionales (familiares, de gemelos, de adopción y combinados), la heredabilidad ${ }^{1}$ de un carácter. Estimando (a partir de valores fenotípicos, observables y medibles) las varianzas genéticas y ambientales entre individuos, sin identificar genes o ambientes concretos (Plomin et al., 2002). Diversas investigaciones en este campo (Bouchard \& Loehlin, 2001; Sullivan \& Kendler, 1999; Sullivan, Kendler, \& Neale, 2003) han dado cuenta que los genes contribuyen a la variación poblacional de caracteres psicológicos. Estudios de adopción realizados en Finlandia (Tienari, Wynne, \& Moring, 1994) y estudios familiares realizados en Dinamarca (Kety, Wender, \& Jacobsen, 1994) mostraron claras evidencias de influencia genética en la esquizofrenia. Sin embargo, la heredabilidad para el trastorno es del $50-60 \%$, por lo tanto, otros factores diferentes a los genes deben desempeñar un papel importante a la hora de determinar 
2 El imprinting genético corresponde a genes que se expresan exclusivamente en uno de los cromosomas parentales, la pérdida del cromosoma $\mathrm{X}$ que contiene los alelos no inactivados da como resultado una ausencia completa de los genes correspondientes.

3 El $50 \%$ de las mujeres con ST presentan ausencia completa del cromosoma X, el $40 \%$ presentan mosaicismo (dos o más líneas celulares), y el $10 \%$ anomalías estructurales del cromosoma $\mathrm{X}$. su inicio (O'Donovan \& Owen, 1999). Asimismo, la heredabilidad estimada para la habilidad cognitiva general (g) es del 52\% (Plomin et al., 2002) y para la personalidad es del 30-50\% (Johnson, McGue, \& Krueger, 2005). Si bien estos resultados proporcionan una prueba indiscutible de la existencia de influencia genética sobre caracteres psicológicos, también proporcionan la mejor evidencia acerca de la importancia de los factores ambientales.

Rutter (2007) considera que los hallazgos en Genética del comportamiento tienen implicancias tanto para la investigación genética como para la psicosocial: "para la genética, el mensaje es que parte del efecto genético se debe en realidad a su impacto indirecto sobre variaciones en la exposición a riesgos ambientales. Para la investigación psicosocial el mensaje es paralelo: algunos de los efectos que parecen ser enteramente ambientales están, en realidad, mediados parcialmente por los genes". Por tanto, la investigación en este campo debe analizar los procesos relacionados con la forma en que los individuos interactúan con su entorno, y con las vías indirectas por las que susceptibilidades genéticamente influenciadas conducen a conductas particulares.

\section{¿Por qué el Síndrome de Turner?}

El Síndrome de Turner es un trastorno cromosómico no heredable, determinado por la delección total o parcial del cromosoma $X$ en el sexo femenino. Su incidencia poblacional es de 1:1900 mujeres nacidas vivas, sin embargo la frecuencia de aparición prenatal es aún mayor a la expuesta, ya que se estima que un $99 \%$ de los embarazos que portan esta anomalía terminan en abortos espontáneos durante el primer trimestre (Murphy, Mazzocco, Gerner, \& Henry, 2006). Si bien el fenotipo externo es muy variable, ciertos rasgos físicos presentan un mayor índice de prevalencia poblacional, tales como: talla baja $(100 \%)$, infertilidad (99\%), ausencia de la maduración puberal (96\%) y cardiopatía congénita (55\%) (Ramos, 2003).

Las nuevas tecnologías en biología molecular establecen relaciones entre el cuadro clínico observado en esta población y la deleción e inactivación ${ }^{2}$ del material genético del cromosoma $X$, relacionando regiones cromosómicas críticas y sus genes correspondientes con determinadas alteraciones físicas y psicológicas. Un ejemplo de lo expuesto lo constituye la talla, cuando la deleción se presenta en el brazo corto (p) la mujer con Síndrome de Turner presentará talla baja. Sin embargo, cuando la deleción se produce en el brazo largo (q), no presentará dificultades en su talla pero tendrá disgenesia gonadal (Bondy, 2003).

En este sentido, diversas investigaciones (Bondy, 2006) han correlacionado la variabilidad genotípica del síndrome ${ }^{3}$ con la variabilidad observada en el fenotipo, dando cuenta que la intensidad y frecuencia de las anomalías somáticas y de las características psicológicas están determinados, en parte, por el grado de deleción y la inactivación o silenciamiento de genes. Sin embargo, estos mismos estudios, reportan diferencias individuales en mujeres con el mismo genotipo, aportando evidencias de la acción del ambiente en la expresión génica.

\section{Aspectos neuropsicológicos en el Síndrome de Turner: interacción genes-ambiente}

La literatura revela que las mujeres con Síndrome de Turner no presentan dificultades 
Sin embargo, se han reportado dificultades en diversos procesos de aprendizaje debido a problemas en el funcionamiento ejecutivo, especialmente en memoria de trabajo y tareas que requieren control de la impulsividad y automonitoreo

(Ross, Roeltgen, \& Zinn, 2006). en las habilidades verbales y las capacidades intelectuales (sólo el $10 \%$ poseen cierto grado de retraso mental o trastornos del lenguaje). Sin embargo, se han reportado dificultades en diversos procesos de aprendizaje debido a problemas en el funcionamiento ejecutivo, especialmente en memoria de trabajo y tareas que requieren control de la impulsividad y automonitoreo (Ross, Roeltgen, \& Zinn, 2006). Asimismo, se han observado déficit en las habilidades visuoespaciales, en tareas visuomotoras y tareas de manipulación de información relación-espacial; registrándose dificultades para generar y/o comprender conceptos abstractos, para planificar y llevar a cabo tareas complejas y mantener la atención activa (Del Alamo, 2006).

La amplia variabilidad en las características neuropsicológicas dentro de esta población impide establecer un único perfil. A este respecto, Mazzocco (2006) considera que, si bien existe consenso en los reportes científicos, esto no significa que todas las mujeres con Síndrome de Turner van a expresar el mismo fenotipo o que todas aquellas que tengan un perfil neuropsicológico similar tendrán grados comparables de fortalezas y debilidades.

Teniendo en cuenta la amplia variabilidad genotípica del síndrome, ciertos descubrimientos científicos apoyan la hipótesis de que la variabilidad en el perfil neuropsicológico podría deberse a la interacción genes-ambiente. Uno de los hallazgos que contribuye al conocimiento de la asociación entre características neuropsicológicas y factores genéticos es la localización molecular de una deleción específica del cromosoma X: Xp22.3. Las mujeres con Síndrome de Turner portadoras de esta deleción expresan un perfil neuropsicológico diferente al observado en mujeres con el mismo síndrome pero con distintos cariotipos (Zinn et al., 2007). A este respecto, Ross et al. (2006) sostiene que la haploinsuficiencia de genes en el brazo corto del cromosoma X es responsable de gran parte del fenotipo cognitivo de las mujeres con Síndrome de Turner. Asimismo, la severidad de las dificultades en el funcionamiento ejecutivo, las habilidades visuoespaciales, visuoperceptuales y habilidades atencionales también parecen estar relacionadas con el cariotipo, siendo menor el rendimiento en mujeres con línea Turner pura $\left(45, x_{0}\right)$ que en aquellas que poseen un cariotipo mosaico con una línea normal $(46, X X / 45, X 0)$ (Ross et al., 2006; Zinn et al., 2007).

Una de las vías de análisis que permite comprender las conexiones entre los genes y la expresión de características neuropsicológicas es el estudio del desarrollo cerebral. En el Síndrome de Turner, los principales hallazgos dentro de esta línea revelan la presencia de una organización cortical y una morfología cerebral atípica, con una alteración en el funcionamiento de cortex prefrontal y una reducción en el volumen del hipocampo y de los lóbulos occipital y parietal superior y anterior (Brown, et al., 2004; Kesler, et al., 2004). Asimismo se ha reportado un agrandamiento en el volumen de la amígdala (Kesler, et al., 2004).

Estas alteraciones en el desarrollo cerebral podrían explicar el deficit neurocognitivo, especialmente en la memoria y en el procesamiento ejecutivo, visuomotor y visuoespacial (Haberecht et al., 2001; Hart, Davenport, Hooper, \& Belger, 2006; Tamm, Menon, Allan, \& Reiss, 2003). 
En concordancia con lo expuesto, la investigación realizada por Monereo Megias y Peñalver Talavera (2003) demostró alteraciones en el desarrollo cerebral de mujeres con Síndrome de Turner, los autores refieren hipodesarrollo de la zona hipocampal y del lóbulo temporal, adjudicando la causa de estas anomalías no sólo al cariotipo sino también a factores ambientales como la deficiencia estrogénica. Estudios realizados por Ross et al. (2003) revelaron que los tratamientos hormonales con estrógeno mejoran la memoria y la capacidad de reacción, y que la administración de oxandrolona actúa sobre la memoria de trabajo, observándose cambios positivos a los dos años de tratamiento.

Cabe destacar, también, la importancia del ambiente familiar y social en el desarrollo neuropsicológico de las mujeres con Síndrome de Turner. Un contexto estimulante resulta propicio para el desarrollo cognitivo general potenciando al máximo sus capacidades. Portellanos (2001) considera que la estimulación temprana resulta relevante ya que la plasticidad cerebral es inversamente proporcional a la edad.

Las investigaciones presentadas refuerzan la hipótesis que considera al Síndrome de Turner como un "modelo genético sensible" donde la expresión diferencial en las características neuropsicológicas surge de la interacción, durante el desarrollo pre y postnatal, de factores genéticos y ambientales.

\section{La disfunción social como variable susceptible de riesgo ambiental en el Síndrome de Turner}

La dificultad de las personas con Síndrome de Turner para interactuar con sus pares es una característica ampliamente descripta en la literatura. En general presentan problemas para constituir nuevas relaciones, conformar y mantener relaciones de amistad y establecer pareja (Danielewicz \& Pisula, 2005; Lesniak Karpiak, Mazzocco, \& Ross, 2003).

Durante el desarrollo atraviesan diversas situaciones disruptivas, consecuencia de las características físicas y psicológicas particulares del síndrome. Según Lesniak Karpiak et al. (2003) en la temprana infancia las relaciones sociales están menos estigmatizadas y dañadas que en la adolescencia. Diversas investigaciones (Danielewicz \& Pisula, 2005; Suzigan et al., 2004; McCauley, Ross, \& Kushner, 1995; Schmidt, Rubinow, \& Bondy, 2006) demostraron menor autoestima, inmadurez emocional y ansiedad social en adolescentes con Síndrome de Turner, en comparación con sus pares de la misma edad. Boman, Möller y Albertsson Wikland (2000) consideran que esto se debe a que las adolescentes son más capaces de comprender las consecuencias negativas del síndrome. El significado que le atribuyan va a depender de su condición médica, y de factores asociados de fuerte impacto emocional como la posibilidad de inferir futuras consecuencias biológicas, psicológicas y sociales para su vida.

La baja talla y el retraso puberal (características físicas que resultan de la expresión génica), son algunos de los factores que podrían estar asociados a los grandes disturbios emocionales y sociales que se observan en adolescentes con Síndrome de Turner (Kagan Krieger, 2001). Brinch y Manthorpe (1987) propusieron la baja talla como el factor de mayor impacto emocional y origen de muchos de los problemas observados. Por otro lado Skuse, Percy y Stevenson (1994) 
consideraron que la asociación entre baja estatura y maduración lenta podría ser la responsable de gran parte de las dificultades psicológicas.

El tratamiento hormonal estrogénico (acción ambiental) y su consecuente inducción de la pubertad desempeñan un papel importante en el desarrollo y madurez emocional. Los estudios de McCauley y Sybert (2006), Boman et al. (2000), Ross, Roeltgen, Feuillan, Kushner y Cutler (1998) y Ross y Roeltgen (1996) demostraron que los efectos del tratamiento estrógenico sobre el funcionamiento psicológico (tanto socioemocional como cognitivo) son positivos y promueven la autopercepción y autoestima.

El retraso puberal podría afectar el funcionamiento psicosocial al menos de dos maneras. La deficiencia hormonal puede impactar en el desarrollo cerebral afectando regiones implicadas en la regulación del afecto y el comportamiento social. Y los factores de la experiencia, asociados con la pérdida de la fertilidad, pueden impactar en la autoestima de las mujeres y en la percepción de su rol en las relaciones (Schmidt et al., 2006; Suzigan, Silva, \& Maciel-Guerra, 2005). Sin embargo, no existe consenso sobre el tiempo de inducción puberal. Por un lado, el desarrollo sexual en concordancia con sus parejas es preferible por razones psicológicas (autoestima, ansiedad social, estrés, etc.) y por el otro lado, los tratamientos estrogénicos reducen la talla final (Boman, Bryman, \& Möller, 2004).

La mayoría de las investigaciones consideran que, si bien la inducción puberal podría establecerse entre los 12 y 15 años, es necesario no sólo tener en cuenta características físicas como: talla, edad ósea, densidad mineral de los huesos; sino también el perfil psicosocial de cada niña (Carel et al., 2005). En este punto, es necesario considerar la importancia que tiene en el desarrollo de la propia imagen corporal el inicio del tratamiento sustitutivo con estrógenos en el momento adecuado. Según Carel et al. (2006), la pubertad debería ser inducida a una edad psicológica apropiada para optimizar la autoestima y el ajuste social. Este modelo de abordaje tiene en cuenta no sólo características físicas, sino también características psicológicas que surgen de la interacción de los genes con factores ambientales susceptibles de modificación.

Otro factor que se ha comenzado a investigar, como posible responsable de la vulnerabilidad social, es el déficit en los procesos cognitivos que subyacen a la interacción social. Según Molko et al. (2004) las dificultades sociales pueden originarse en un déficit de la lectura de la información socialmente relevante. En una revisión realizada por Mazzocco, en el año 2006, se propone que los problemas en el funcionamiento social pueden ser secundarios a disfunciones en otras áreas como déficit en el procesamiento afectivo/facial, dificultades en la expresión del lenguaje, o a un repertorio limitado de comportamientos sociales. En esta línea, el punto central de análisis es el estudio de la cognición social como variable mediadora entre el funcionamiento cognitivo y la interacción social, intentando relacionar factores genéticos y ambientales. La cognición social es el conjunto de operaciones mentales que subyacen en las interacciones sociales, e incluyen los procesos implicados en la percepción, interpretación y generación de respuestas ante las intenciones, disposiciones y conductas de otros (Brothers, 1990). 
Corresponde a los procesos cognitivos implicados en cómo la gente piensa sobre ella misma, otras personas, situaciones sociales e interacciones (Penn, Corrigan, Bental, Racenstein, \& Newman, 1997).

Uno de los primeros estudios realizados en cognición social en el Síndrome de Turner es el de Skuse et al. (1997) cuyo objetivo fue establecer la relación entre impronta genómica y cognición social. Sus resultados demostraron que las mujeres que heredan el cromosoma X materno presentan mayores dificultades en esta área que aquellas que heredan el $X$ paterno. Según Skuse hay un locus genético para la cognición social, que es imprintado y se expresa por la herencia del cromosoma materno. Asimismo, Lawrence et al. (2007) reportó que las mujeres con monosomía del $\mathrm{X}$ donde el cromosoma $\mathrm{X}$ es heredado de la madre tienen mayores dificultades en el ajuste social que aquellas que heredan el cromosoma paterno.

Otros estudios han intentado especificar las consecuencias de la perdida del cromosoma $X$ en la anatomía del cerebro y su relación con los procesos de la cognición social. Como fuera expuesto, Kesler et al. (2004) reportó alteraciones en el volumen de la amígdala y el hipocampo, y Molko et al. (2004) cambios macroscópicos en regiones corticales específicas tales como el giro temporal superior izquierdo, el cortex orbitofrontal, y el giro intraparietal derecho. Ambos autores relacionaron estas alteraciones en el desarrollo del cerebro con disfunciones en los procesos de cognición social.

La investigación de la cognición social en el Síndrome de Turner constituye otro de los modelos que intenta comprender las vías que conectan los genes con la conducta.
Este modelo pretende explicar la disfunción social que presentan las mujeres con este síndrome relacionando expresión génica, desarrollo cerebral y conducta.

\section{Consideraciones finales}

El análisis precedente da cuenta de como las particularidades genéticas, físicas y psicológicas que expresan las mujeres con Síndrome de Turner afectan la capacidad y calidad de sus relaciones sociales. Si bien en la infancia las dificultades en el aprendizaje solapan esta disfunción, en la adolescencia se vuelve más pronunciada teniendo repercusiones en la edad adulta. Los modelos propuestos para el estudio de la disfunción social indagan sobre la forma en que actúa el ambiente sobre el desarrollo psicológico, y de cómo a su vez éste se ve afectado por factores genéticos que actúan en interacción.

Identificar mecanismos de riesgo ambiental, tales como, falta de estimulación temprana, retraso en la inducción puberal, escaso apoyo social, experiencias educativas y familiares negativas, etc., que repercuten a nivel psicológico y potencian la disfunción social en una población vulnerable como las mujeres con Síndrome de Turner, permite el diseño de programas de prevención e intervención psicológica en el ámbito individual y familiar. Estos programas favorecerán el fortalecimiento y generación de nuevos recursos en las mujeres con Síndrome de Turner, potenciando la calidad de sus interacciones, la confianza en sí mismas y en sus capacidades para comunicarse y vincularse.

Asimismo, el desarrollo de nuevas técnicas de investigación como la genética molecular, la neuroimagen funcional y el estudio 
de la bioquímica cerebral, así como la incorporación de un equipo de trabajo multidisciplinario (neurólogos, pediatras, genetistas, psicólogos, docentes y padres) al estudio de los trastornos genéticos como el
Síndrome de Turner, permite un abordaje integral, desde un enfoque biopsicosocial, y un mayor conocimiento y comprensión de la interacción entre los genes y el ambiente en la expresión de la conducta. 


\section{Marcela Carolina López*}

Licenciada en Ciencias Biológicas. Docente - investigador de la Facultad de Psicología, Universidad Nacional de Mar del Plata

\section{María Jose Aguilar}

Becaria de la Universidad Nacional de Mar del Plata, Facultad de Psicología

*Funes 3250, Cuerpo V- Nivel III, Mar del Plata (7600), Bs. As., Argentina

E-mail: mclopez@mdp.edu.ar

Recebido 16/07/2008 Reformulado 03/02/2009 Aprovado 16/02/2009

\section{Referencias}

Artigas Pallarés, J., Gabau Vila, E., \& Guitart Feliubadaló, M. (2006). Fenotipos conductuales en el retraso mental de origen genético. Revista de Neurología, 42(1), 15-19.

Bleichmar, E. (2004). Modelos interactivos entre la genética de la conducta y la parentalización. Revista de Psicoanálisis, 17. Recuperado el 17 de abril del 2008, de http://www.aperturas. org/articulos

Boman, U., Bryman I., \& Möller, A. (2004). Psychological wellbeing in woman with Turner syndrome: Somatic and social correlatos. Journal of Psychosomatic Obstetrics \& Gynecology, $25,211-219$.

Boman, U., Möller, A., \& Albertsson Wikland, K. (2000). Selfperception, behavior and social functioning in Swedish girls with Turner syndrome: A population-based study. Göteborg Psychological Reports, 30(5), 1-12.

Bondy, C. (2003). Turner syndrome: Genotype \& phenotype. Recuperado el 4 de octubre del 2007, de http:// turners.nichd. nih.gov/TS_Protocol_00-CH-0219.pdf

Bondy, C. (2006). Care of girls and women with Turner syndrome: A guideline of the Turner syndrome study group. Journal of Clinical Endocrinology \& Metabolism, 92(1), 10-25.

Bouchard, T., \& Loehlin, J. (2001). Genes, evolution, and personality. Behavior Genetics, 31(3), 243-273.

Brinch, M., \& Manthorpe, T. (1987). Short stature as a possible etiological factor in anorexia nervosa. Acta Psychiatrica Scandinavica, 76, 328-32.

Brothers, L. (1990). The social brain: A project for integrating primate behaviour and neurophysiology in new domain. Concepts in Neuroscience, 1, 27-61.

Brown, W., Kesler, S., Eliez, S., Warsofsky, I., Haberecht, M., \& Reiss, A. (2004). A volumetric study of parietal lobe subregions in Turner syndrome. Developmental Medicine \& Child Neurology, 46(9), 607-609.

Carel, J., Ecosse, E., Bastie Sigeac, I., Cabrol, S., Tauber, M., Léger, J. et al. (2005). Quality of life determinants in young women with Turner's syndrome after growth hormone treatment: Results of the StaTur population-based cohort study. The Journal of Clinical Endocrinology \& Metabolism, 90(4), 1992-1997.
Carel, J., Elie, C., Ecosse, E., Tauber, M., Léger, J., Cabrol, S. et al. (2006). Self-esteem and social adjustment in young women with Turner syndrome-influence of pubertal management and sexuality: Population-based cohort study. Journal of Clinical Endocrinology \& Metabolism, 91(8), 2972-2979.

Danielewicz, D., \& Pisula, E. (2005). Self-eteem evaluation of girls of Turner syndrome. Annales Universitatis Mariae CurieSkłodowska Lublin-Polonia, 60(16), 72.

Del Alamo, A. (2006). Problemas psicológicos y neuropsicológicos del síndrome de Turner. Recuperado el 9 de abril del 2008, de www.psicología-online.com/articulos/2006/Sindrome_turner

Haberecht, M., Menon, V., Warsofsky, I., White, C., Dyer Friedman, J., Glover, G. et al. (2001). Functional neuroanatomy of visuo-spatial working memory in Turner syndrome. Human Brain Mapping, 14(2), 96-107.

Hart, S., Davenport, M., Hooper S., \& Belger A. (2006) Visuospatial executive function in Turner Syndrome: Functional Mri and neurocognitive findings. Brain, 129(5), 1125-1136.

Johnson, W., McGue, M., \& Krueger, R. (2005). Personality stability in late adulthood. A behavioral genetic analysis. Journal of Personality, 73(2), 523-525.

Kagan Krieger, S. (2001). Factors that affect coping with Turner. Syndrome Journal of Nursing Scholarship, 33(1), 43-45.

Kandel, E. (2000). Principios de neurociencias. Madrid: McGrawHill.

Kesler, S., Garrett, A., Bender, B., Yankowitz, J., Zengd, S., \& Reiss, A. (2004). Amygdala and hippocampal volumes in Turner syndrome: A high-resolution MRI study of X-monosomy. Neuropsychologia, 42(14), 1971-1978.

Kesler, S., Haberecht, M., Menon, V., Warsofsky, I., DyerFriedman, J., Neely, J., \& Reiss, A. (2004). Functional neuroanatomy of spatial orientation processing in Turner syndrome. Cerebral Cortex, 14(2), 174-180.

Kety, S., Wender, P., \& Jacobsen, B. (1994). Mental illness in the biological and adoptive relatives of schizophrenic adoptees: Replication of the Copenhagen study in the rest of Denmark. Archives of General Psychiatry, 51, 442-455.

Lawrence, K., Jones, A., Oreland, L., Spektor, D., Mandy, W. Campbell, R., \& Skuse, D. (2007). The development of mental 
state attributions in women with X-monosomy, and the role of monoamine oxidase $B$ in the sociocognitive phenotype. Cognition, 102(1), 84-100.

Lesniak Karpiak, K., Mazzocco, M., \& Ross, J. (2003). Behavioral assessment of social anxiety in females with Turner or fragile $\mathrm{X}$ syndrome. Journal of Autism and Developmental Disorders, 33(1), 55-67.

Mazzocco, M. (2006). The cognitive phenotype of Turner syndrome: Specific learning disabilities. International Congress Series, 1298, 83-92

McCauley, E., Ross, J., \& Kushner, H. (1995). Self-esteem and behaviour in girls with Turner syndrome. Developmental and Behavioral Pediatrics, 16, 82-88.

McCauley, E., \& Sybert, V. (2006). Social and behavioral development of girls and women with Turner syndrome. International Congress Series, 1298, 93-99.

Molenaar, P., Boomsma, D., \& Dolan, C. (1993). A third source of developmental differences. Behavior Genetics, 23(6), 519-531.

Molko, N., Cachia, A., Rivière, D., Mangin, J., Bruandet, M. \& LeBihan, D. (2004). Brain anatomy in Turner syndrome: Evidence for impaired social and spatial-numerical networks. Cerebral Cortex, 14, 840-850.

Monereo Megias, S., \& Peñalver Talavera, D. (2003). La muje adulta con síndrome de Turner: algunas consideraciones. In Sociedad Española de Endocrinología Pediátrica (Orgs.), Síndrome de Turner (pp. 147-165). Barcelona: J \& C Ediciones Médicas S. L.

Murphy, M., Mazzocco, M., Gerner, G., \& Henry, A. (2006) Mathematics learning disability in girls with Turner syndrome or fragile X syndrome. Brain and Cognition, 61(2), 195-210.

O'Donovan, M., \& Owen, M. (1999). Candidate-gene association studies of schizophrenia. American Journal of Human Genetics, 65, 587-592.

Penn, D., Corrigan, P., Bentall, R., Racenstein, J., \& Newman L. (1997). Social cognition in schizophrenia. Psychological Bulletin, 121, 114-132.

Plomin, R., \& Crabbe, J. (2000). DNA. Psychological Bulletin, 126(6), 806-828.

Plomin, R., DeFries, J., McClearn, G., \& McGuffin, P. (2002). Genética de la conducta. Buenos Aires: Ariel Ciencia.

Plomin, R., Owen, M., \& McGuffin, P. (2004). The genetic basis of complex human behaviors. Science, 264 (5166), 1733-1739.

Portellanos, J. (2001). Aspectos neuropsicológicos del síndrome de Turner. In M. Pombo \& E. Vicens, El síndrome de Turner. Enfoque multidisciplinario y optimización del tratamiento con GH (pp. 91-96). Pharmacia Spain.

Puertas, M. (1992). Genética, fundamentos y perspectivas. Caracas: Interamericana; McGraw-Hill.

Ramos, F. (2003). Aspectos clínicos-epidemiológicos. In Sociedad Española de Endocrinología Pediátrica (Org.), Síndrome de Turner (pp. 1-12). Barcelona: J \& C Ediciones Médicas S. L.

Ross, J., \& Roeltgen, D. (1996). Self-concept and behavior in adolescent girl with Turner syndrome: potencial estrogen effects. Journal of Clinical Endocrinology \& Metabolism, 81 926-931.

Ross, J., Roeltgen, D., Feuillan, P., Kushner, H., \& Cutler, J. (1998). Effects of estrogens on nonverbal processing speed and motor functions in girls with Turner's syndrome. Journal of Clinical Endocrinology \& Metabolism, 83, 3198-3204.
Ross J., Roeltgen, D., Stefanatos, G., Feuillan, P., Kushner, H., Bondy, C., \& Cutler, J. (2003). Androgen-responsive aspects of cognition in girls with Turner syndrome. Journal of Clinical Endocrinology \& Metabolism, 88(1), 292-296.

Ross, J., Roeltgen, D., \& Zinn, A. (2006). Cognition and the sex chromosomes: Studies in Turner syndrome. Hormona Researchs, 65, 47-56.

Rutter, M. (2007). Naturaleza vs. ambiente. Recuperado el 20 de marzo del 2008, de http://www.portalplanetasedna.com. ar/ciencia02.htm

Schmidt, P., Rubinow, D., \& Bondy, C., (2006). Adult women with Turner syndrome: A systematic evaluation of current and past psychiatric illness, social functioning, and self-esteem. International Congress Series, 1298, 100-107.

Skuse, D., Percy, E., \& Stevenson, J. (1994). Psychosocial functioning in the Turner syndrome: A national Surrey. In B. Stabler \& L. Underwood (Eds.), Growth, stature and adaptation. behavioral, social and cognitive aspects of growth delay (pp. 15-64). Chapel Hill: The University of North Carolina.

Sullivan, P., \& Kendler, K. (1999). The genetic epidemiology of smoking. Nicotine \& Tobacco Research, 1(Suppl. 2), 51-57.

Sullivan, P., Kendler, K., \& Neale, M. (2003). Schizophrenia as a complex trait evidence from a meta-analysis of twin studies. Archives of General Psychiatry, 60, 1187-1192.

Suzigan, L. Z. C., Silva, R. B. P., \& Maciel-Guerra, A. T. (2005). Aspectos psicossociais da Síndrome de Turner. Arquivos Brasileiros de Endocrinologia \& Metabologia, 49(1), 157-164.

Suzigan, L. Z. C., Silva, R. B. P., Marini, S. H. V. L., Baptista, M. T. M., Guerra Jr., G, Magna, L. A., \& Maciel-Guerra, A. T. (2004). A percepção da doença em portadoras da síndrome de Turner. Jornal de Pediatria, 80(4), 309-314.

Suzuki, D., Griffiths, A., Miller, J., \& Lewontin, R. (1996). Introducción al análisis genético. Editorial Interamericana: McGraw-Hill.

Tamm, L., Menon, V., Allan L., \& Reiss, A. (2003). Abnormal prefrontal cortex function during response inhibition in Turner Syndrome: Functional magnetic resonance imaging evidence. Society of Biological Psychiatry, 53(2), 107-111.

Tienari, P., Wynne, L., \& Moring, J. (1994). The finnish adoptive family study of schizophrenia: Implications for family research. British Journal of Psychiatry, 23(Suppl.), 20-26.

Zinn, A., Roeltgen, D., Stefanatos, G., Ramos, P., Elder, F. Kushner, H., Kowal, K., \& Ross, J. (2007). A Turner Syndrome neurocognitive phenotype maps to Xp22.3. Behavioral and Brain Functions, 3(24), 1-14. 